Gymnadenia conopsea $\times a l b i d a$.

Labellum profunde trifidum obligue descendens lobis subaequis ovatis obtusis; calcar crassum, ovarium quarta parte superans; perigonï lacinia exteriora late patentia, cetera modice conniventia; bracteae ovarium paulo superantia; spica cylindrica subsecunda; folia inferiora ovata obtusa, superiora ovato-lanceolata. acuta; lubera digitata. Color florum roseo-albidus.

\title{
Ein Ausflug in das Bars-Honter Comitat.
}

\section{Von Josef Knapp.}

Quam quam animas meminisse lorre: Lustu gue refugit-encipiam, Virg.

An einem Mitwoch im Monate Juli 1863 verliess ich Lapás-Gyarmath schon in den ersten Morgenstunden und schnellen Schrittes eilte ich der Hauptstrasse zu. Auf dem Wege dahin fand ich an den Ackerrändern Euphorbia exigua, Fumaria Vaillantii, Lactuca saligna, Linaria Elatine, Nonnea pulla, Picris hieracioides und Tordylium maximum. An Wassergräben in der Nähe der Csendes-Puszta sah ich: Epilobium parviflorum, Juncus effusus, lamprocarpus und compressus, Nasturtium palustre, Prunella vulgaris und Triglochin palustre. Auf dem weilern Wege blühten zu beiden Seiten: Adonis aestivalis, Ajuga Chamaepilys, Andropogon Ischaemum, Asperula cynanchica, Cirsium eriophorum, Echium vulgare, Fragaria vesca, Medicago sativa verwildert, Melampyrum barbatum, Nigella arvensis, Ononis spinosa, Poa compressa, Potentilla argentea, Poterium Sanguisorba, Raphanus Raphanistrum und Scabiosa ochroleuca.

Auf der Strasse angelangt, hatte ich zu beiden Seiten Waldungen, bestehend aus Quercus pedunculata und pubescens, unter welchen Crataegus oxyacantha, Rosa canina und Evonymus europaeus als Unterholz vorkommen. Gleich am Errde des Waldes breitet sich zur linken Seite eine kleine Wiese aus, daselbst fand ich Caltha palustris, Convolvulus sepium, Juncus effusus und Lysimachia nummularia. Weiterhin bis zum Grenzsteine des Unter-Neutraer Comitates war die Vegetation auf die neben der Strasse sich hinziehenden Gräben beschränkt. Agrimonia Eupatoria, Amaranthus retroflexus, Carex muricata, Hieracium Auricula, Lepidium ruderale, Marrubium peregrinum, Podospermum Jacquinianum. Potentilla argentea, Stachys palustris und Verbena officinalis traf ich daselbst an. Weiter gegen Verebély war die Flora wenig überraschend und nur ein vor Verebély vereinzelt dastehendes Banernhaus fesselte meine Aufmerksamkeit. Hinter dem Garten des genannten Hauses breitet sich Alluvialterrain aus, Angelica sylvestris, Carex hirta, Cyperus fuscus, Erythraea pulchella, Gnaphalium uliginosum, Gratiola officinalis, Juncus bufonius, lamprocarpus, effusus, Lactuca saligna, Lythrum Salicaria, Myosotis palustris, Potentilla supina, Tanacetum vulgare, Veronica Anagallis 
und Beccabunga legte ich daselbst ein. Nächst dem Wege von Verebély nach Tild befinden sich Wassergräben, Juncus effusus, lamprocarpus, Butomus umbellatus, Galega officinalis, Scirpis maritimus und Sparganium ramosum sammelte ich daselbst. Weiterhin sah ich auf Brachäckern und nächst dem Wege Alyssum calycinum, Astragalus Cicer, Cynodon Dactylon, Echium italicum, Lactuca saligna, Lepidium Draba, Linaria Elatine, spuria, Nigella arvensis, Rubus caesins, Rumex crispus, Salsola Kali, Saponaria officinalis, stachys annua und Trifolium arvense.

Von Tild bis Csiffär fand ich auf Aeckern Ajuga Chamaepitys, Aristolochia Clematitis, Armoracia rustica Fl. d. W., Ballota nigra, Cephalaria transsilvanica, Euphorbia nicaeensis vereinzelt, Farsetia incana, Marrubium peregrinum, Verbena officinalis, Xanthium strumarium und spinosum. Weiter gegen Ober-Györöd traf ich auf Ackerrändern an: Anthemis Cotula, Artemisia Absynthium, Atriplex nitens an Zäunen bei Csiffár Euphorbia platyphylos, Lamium amplexicaule, Lapsana communis, Linaria Elatine, Salvia silvestris, Senecio viscosus und Spiraea Filipendula an Waldrändern. Am Bache neben der Strasse blühten damals Alisma Plantago, Alopecurus geniculatus, Bulomus umbellatus, Myosotis palustris, Polygonum amphibium, Potamageton erispus, Ranunculus acris, Senecio Jacobaea, Stachys palustris, und Tussilago Farfara. Weiterhin fand ich noch auf Aeckern: Asperula cynanchica, Lepidium campestre, Scleranthus annuus und Stellaria graminea L. Von Ober-Györöd bis Gyökönyes war die Flora interessanter. Gleich in der Nähe des Dorfes befindet sich ein Wäldchen, das sich neben der Strasse dahinzieht: Carex Schreberi, Anthriscus silvestris, Bupleurum Gerardi, Crepis tectorum, Dianthus Armeria, Filago minima, Hypericum perforatum, Potentilla recta, Pioris hieracioides, Rumex Acetosella, Senecio erucifolius, Turritis glabra, Veronica chamaedrys und Verbascum nigrum legte ich daselbst ein. Weiler hin fand ich noch zu beiden Seiten des Weges Agrimonia Enpatoria, Artemisia scoparia, Carduus acanthoides, weissblühend, Cuscuta europaea verheerend auf Luzernefeldern, Erysimum repandum, Nigella arvensis und Stellaria graminea.

Bei Gross-Kállna sah ich am diesseitigen Ufer der Gran: Alnus glutinosa, Eragrostis poaeoides, Mentha aquatica, Juncus bufonius, lamprocarpus, Lathyrus tuberosus, Lepigonum rubrum, Limosella aquatica, Lythrum salicaria, Malachium aquaticum, Melilolus alba, Rubus caesius, Sagina procumbens und Vicia Cracca. Von hier ging ich über die Brücke, um ans jenseitige Ufer zu gelangen. Hier fand ich eine Vegetation wie ich sie bisher nirgends bemerkte, Pflanzen, die sonst klein sind und leicht dem Auge des Floristen entgehen, waren hier in monströsen Exemplaren. Artemisia scoparia, Cerastium arvense. Chenopodium Botrys und polyspermum, Centunculus minimus, Gnaphalium uliginosum, Gypsophila muralis, Herniaria glabra, Scirpus Holoschoenus, Limosella aquatica, Lythrum hyssopifolium, Nasturtium palustre, Oenanthe Phellandrium, Potentilla 
supina, Ranunculus sceleratus, Sagina procumbens, Scirpus acicularis, Tussilago Farfara, Veronica Beccabunga und serpyllifolia traf ich hier an. Weiter gegen Szécs war Armoracia rustica, Artemisia scoparia vereinzelt und Aristolochia Clematitis häufig auf Hanffeldern. Gegen Léva hin bestand die ganze Pflanzendecke vorzugsweise aus Polygonum aviculare und erst auf den Wiesen fand ich Gratiola officinalis.

In Léva beschloss ich die Nacht zu verbringen, um so am nächsten Morgen meine botanischen Streifzüge fortselzen zu können.

Am folgenden Tage besichtigte ich den Markt und die alte Schlossruine, an welche sich viele historische Erinnerungen knüpfen, die aber zu erzählen die Kürze des Orles nicht gestattet. Im Innern des Marktfleckens fand ich Artemisia scoparia, vulgaris, Absynthium, Asperugo procumbens, Ballola nigra. Bryonia alba, Cynoglossum officinale, Lepidium Draba, Nepeta Cataria, Portulacca oleracea, Silene inflata, Verbena officinalis, Xanthium spinosum. Auf der Schlossruine kam Sempervivum hirtum, Reseda lutea und luteola und Stachys recta L. vereinzelt vor. Auf den Wiesen traf ich an: Alopecurus pratensis, Bidens tripartita, Carum Carvi, Cichorium Intybus, Cirsium canum, Dipsacus silvestris, Echinops sphaerocephalus, Galium verum, Humulus Lupulus, Inula salicina, Lythrum salicaria, Nasturtium silvestre, Ranunculus acris, repens, Senecio Jacobaea, Tragopogon pratensis und Veronica serpyllifolia.

Am Bache blühten: Achillea nobilis, Arenaria serpyllifolia, Carex muricata, Clematis integrifolia, Euphorbia Esula, Galego officinalis; Gnaphalium uliginosum, Heracleum Sphondylium, Juncus effusus, lamprocarpus, bufonius, oompressus, Lathyrus pratensis, tuberosus, Linum catharticun, Lysimachia vulgaris, Lythrum hyssopifolium, Hentha aquatica, silvestris, Oenanthe Phellandrium, Polygonum amphibium, Scrophularia aquatica, nodosa, Sparganium ramosum, Triglochin palustre, Erythraea pulchella, Gypsophila muralis, Glyceria fluitans und Cyperus fuscus.

Interessanter war die Flora des Weingebirges. Acer tataricum Arenaria serpyllifolia, Bupleurum Gerardi, Campanula glomerata, Crupina vulgaris in der Nähe des Triangularzeichens nächst der Hauptstrasse nach Benedek, Inula ensifolia, Orchis Morio, Poterium Sanguisorba, Scabiosa transsilvanica, Sedum acre, Valeriana officinalis legte ich daselbst ein.

Am 28. Juli unternahm ich einen Ausflug nach Szántó. Den Weg dahin wählte ich durch das Léva'er Weingebirge und als ich ausserhalb Lèva war, da fand ich auf angeschwemmten Wiesen Geranium palustre, Rumex maritimus, Scirpus maritimus.

Auf den Aeckern waren häufig verbreitet: Anthemis Colula, Cerastium arvense, Chondrilla juncea, Crespis setosa, Euphorbia platyphyllos, Geranium pusillum, Heliotropium europaeum, Lactuca saligna, Nigella arvensis, Prunella vulgaris.

Im W eingebirge legte ich ein: Agrimonia Eupatoria, Asparagus officinalis, Gupleurum Gerardi, Campanula glomerata, Carlina vul- 
garis, Chelidonium majus, Chenopodium polyspermum, Chrysanthemum corymbosum, Cornus sanguinea, Cucubalus bacciferus in Gebüschen sich linanwindend, Cynanchum Vincetoxicum, Cytisus nigricans, Dianthus Armeria, collinus W.K. Erysimum canescens, $\mathrm{Fe}^{\prime}$ stuca inermis, Galium crucialum, Geranium sanguineum, Hieracium umbellatum, Lathyrus pratensis, platyphyllos, Ligustrum vulgare, Melampyrum nemorosum, Origanum vulgare, Orobus niger, Peucedanum Cervaria, Ranunculus lanuginosus, Rosa gallica, Salvia pratensis, verticillata, Scrophularia nodosa, Silene mutans, inflata, Stachys Betonica B enth., Tordylium maximum, Verbascum Blattaria, nigrum und Veronica chamaedrys.

Auf dem Wege von Vársány bis Kiskér fand ich Salvia verticillata, Atriplex nitens, Erysimum canescens, Lacluca Scariola.

Bei Kiskér bemerkte ich Humulus Lupulus, Leonurus Cardiaca, Lepidium Draba, Malva rotundifolia, Marrubium peregrinum und Senecio Jacobaea.

Von hier nahm ich den Weg durch die dortigen Wiesen und gelangte zu einem Bächlein.

Hier kamen häufig vor: Festuca elatior, Juneus compressus lamprocarpus, effusus. Lemna minor, Lycopus europaeus, Poa Eragrostis, Ranunculus aquatilis, bulbosus, sceleratus, Scirpus palustris und Sium latifolium.

Von diesem Bache kam ich zur Hauptstrasse, die nach Száutó führt. Neben der Strasse an dem Graben traf ich Hibiscus Trionum zerstreut an. Weiterhin dehnten sich zur rechten Seite geräumige Waldungen und Holzschläge mit einer günstigen Flora aus. Achillea nobilis, Antirrhinum Orontium, Arenaria serpyllifolia, Artemisia Absynthium, Astragalus glycyphyllos, Calaminiha Acinos, Campanula glomerata, Carex digitata, Chenopodium polysperinum, Clinopodium vulgare, Cynanchum Vincetoxicum, Cytisus nigricans, Dianthus Armeria, Digitalis grandiflora var. ochroleuca, Epilobium angustifolium, montanum, Erythraea Centauriam, Gnaphalium sylvaticum, uliginosum, Lactuca Scariola, Lapsana communis, Lavatera thuringiaca. Linaria Elatine, Origanum vulgare, Prunella vulgaris, $\mathbf{S} a-$ gina procumbens, Scleranthus annuus, Sedum acre, Seseli coloratum, Senecio viscosus, Silene vespertina, inflata, Solanum nigrum, Tanacetum vulgare, Verbascum nigrum, Veronica officinalis, serpyllifolia, spicata, Vicia pisifomis, Violu odorata, Crepis foetida, Euphorbia amygdalioides, Genista tinctoria Geranium Robertianum und Gypsophila muralis waren die Repräsentanten der dorligen Flora.

Von hier gegen Szántó hin bemerkte ich noch auf Aeckern Cephalaria transsilvanica, Csrysanthemum inodorum, Datura Stramonium, Erigeron acris $\beta$ serotinus, Hieracium Auricula, Lepidium ruderale, Linaria spuria, Marrubium peregrinum, Nonnea pulla, Passerina annua, Ranunculus bulbosus, lanuginosus, Stachys annua und Stellaria graminea.

Von den Aeckern stieg ich hinab auf die Wiesen des Dorfes 
Szánto. Hier fand ich cinen üppigen Graswuchs und die Wiesen Flora reichlicher als die der früheren Ortschaften.

Alisma Plantago, Artemisia Absynthium, Astragalus Cicer am Bächlein, Briza media, Butomus umbellatus. Callitriche vernalis, Caltha palustris, Convolvulus sepium, Crepis setosa, biennis, Epilobium hirsutum, palustre, Erythraea pulchells, Eupatorium Cannabinum, Galega officinalis, Galium rubioides; Glyceria fluitans, Hypericum tetrapterum, Juncus lamprocarpus, Tenageia, Lactuca saligna, Lathyrus tuberosus, pratensis, Lemna minor, Linum catharticum, Lotus corniculatus mit der Varielät tenuifolius, Lysimachia nummularia, vulgaris, Malachium aquaticum, Medicago lupulina, Mentha aquatica, silvestris, Myosotis palustris, Myriophyllum verticillatum, Scirpus lacustris, Scrophularia nodosa, Spiraea Aruncus, Tordilium maximum, Triglochin palustre, Veronica Beccabunga, serpyllifolia, Cyperus fuscus, Sium latifolium, Solanum Dulcamara und Potamogeton crispus waren die Ausbeute, welche ich auf den Wiesen, an den Wassergräben und Zäunen um Szántó gemachl habe.

Doch der Abend nabte heran und überzengt, dass man auf den kleinen Dörfern nur mit grosser Anstrengung und um einen hohen Preis ein bequemes Nachllager bekommt, eilte ich nach Léva zurück, wohin ich erst um 10 Uhr gelangte.

Am folgenden Morgen verliess ich Léva und nahm meinen Weg gegen Báth hin. Auf dem Wege dahin fand ich auf den Aeckern und naheliegenden Wiesen Achillea nobilis, Agrimonia Eupatoria, Alnus glutinosa am Bache, Alopecurus geniculatus am Bache, Artemisia scoparia, Bupleurum Gerardi auf Weinbergtriften, Cephalaria transsilvanica, Crepis foelida, setosa, Cynoglossum officinale, Eriophorum angustifolium auf Sumpfwiesen nuben "Nakov-potok" Erysinum canescens, Erythraea pulchella, Epilobium palustre. Falcaria Rivini. Farsetia incana, Juncus conglomeratus. Am Bache J. lamprocarpus. Auf Sumpfwiesen J. effusus, bufonius. Am Bache Lactuca Scariola, saligna, Lemna minor. Am Bache Linaria spuria, Elatine, Linum catharticum, Lotus corniculatus var. tenuifolius, auf Sumpfwiesen, Marrubium peregrinum, Myosotis palustris. Nigella arvensis, Origanum vulgare, an Waldrändern Poa compressa, Podospermum Jacquinianum, Ranuncuius Flammula, Reseda Phyteuma, Salsola Kali, Tragopogon pratensis, Triglochin palustre und Veronica Beccabunga.

Zwischen Báth und Bagonya blühten noch der Strasse entlang und an Waldrändern Artemisia Absynthium, Cephalaria transsilvanica, Dianthus Armeria, Genista tinctoria, Lepidium campestre, Linaria genistaefolia, Lysimachia vulgaris. Am Bache Origanum vulgare, Saponaria officinalis und Tanacetum valgare.

Auf dem Wege von Bagonya bis Steinbach sammelte ich der Strasse entlang, an den Bergbächen und in den benachbarten Waldungen Alnus glutinosa, Anagallis arvensis, Antirrhinum Orontium, Artemisia Absynthium, Bidens tripartita, Bromus inermis, Campanula glomerata, Carlina valgaris, Clematis Vitalba, Cytis $\because s$ nigri- 
tans, Dianthus Armeria, Carthusianonim, prolifer, Eupatorium cannabinum, Fagus silvatica, Galeopsis Ladanum, Geum urbanum, Herniaria glabra an den Bächen, Hieracium Pilosella, Inula hirta. Juniperus communis, Juncus bufonius. Im Sande der Bäche Lysimachia vulgaris, Myosolis palustris, Origanum oulgare, Polygonum Convolvalus, Potentilla argentea, Prunus spinosa, Salvia verticillata, Scrophularia nodosa, Scutellaria galericulata, hastifolia, Teucrium Chamaedrys, Vlmus campestris, Verbascum nigrum und Veronica spicata.

In den Wäldern von Steinbach bis Sziglisberg traf ich noch folgende Gewächse an: Asarum europaeum, Atriplex nitens, Barbarea rulgaris, Bellis perennis, Betula alba, Briza media, Callitriche vernalis an Wassergräben, Caltha palustris, Campanula bononiensis, persicifolia, Tracheliun, Carlina vulgaris, Chelidonium majus, Chenopodium bonus Henricus, polyspermum, Circaea lutetiana, Clinopodium vulgare, Cornus sanguinea, Cynoglossum officinale, Cylisus nigricans, Dianthus Armeria, Epilobium palustre, Erigeron acris $\beta$. serotinus, Erythraea Centaurium, Euphorbia amygdaloides, Fragaria vesca, Genista tinctoria, Geranium Robertianum, Geum urbanum, Glechoma hederacea, hirsuta, Glyceria fuitans, Gnaphalium silvaticum, uliginosum, Linum catharticum, Lysimachia vulgaris, Myoso(is palustris, Orobus vernus, Ribes grossularia, Rubus frulicosus, Rumex obtusifolius, Sagina procumbens, Salix alba, Sulvia verticillata, Sanicula europaea, Senecio visco; us, erucifolius, Silene inflata, nutans, Spiraea Filipendula, Stachys silvestris, Stellaria graminea, Verbascum nigrum, Veronica Chamaedrys, Viola odorala, Cynanchum Vincetoxicum, Impatiens noli tangere, Inula Conyza D C. Luzula albida, Melampyrum nemorosum, Melica nutans, Ranunculus Flammula, Rumex Acetosella und Scleranthus annuus.

Von hier über Windschacht bis Schemnitz sah ich zu beiden Seiten des Weges Achillea nobilis, Aegopodium Podagraria, Artemisia Absynthiam, Barbarea vulgaris, Chenopodium bonus Henricus, Cynanchun Vincetoxicum, Cynoglossum officinale, Erigeron acris, Galeopsis Tetrahi, Geum urbanum, Potentilla argentea, Rumex Acetosella, Salvia verticillata, Scleranthus annuus, Sedum acre, Teucrium Chamaedrys und Verbascum nigrum.

\section{Zur Flora yon Schemnitz.}

Nachfolgendes Verzeichniss der von mir hier angeführten Pflanzenarten ist das Resultat fünftägiger Ausflüge, welche ich in der Umgebung von Schemnitz gemacht habe. Es macht keineswegs Ansprüche auf Vollkommenheit, hat vielmehr den Zweck, einen kleinen Beitrag zur Kenntniss der Flora dieser Stadt zu geben, da mit Ausnahme jener Mittheilungen, welche Kitaibel, Ungarns hochverdienter Botaniker, in Schultess Oesterreichs Flora, "Iter arvense" ${ }^{1}$ ) and

1) Verhandl. der k. k. zool.-bot. Gesellsch. 1863. 
. Addimenta ad Floram hungaricam edidit A. Kanitz ${ }^{1}$ ) gegeben, meines Wissens Nichts über dieselbe veröffentlicht wurde.

Schliesslich sei es mir noch erlaubt zu bemerken, dass Herr Scholtz, Professor am Lyceum daselbst so gütig war, mich auf meinen Ausflügen zu begleiten, mich auf Manches aufmerksam zu machen, was meinem Auge entgangen wäre, wofür ich ihm hier meinen herzlichsten Dank sage.

Clematis Vitalba an Zäunen und Hecken, Anemone pratensis, Pulsatilla auf Bergtriften, silvestris in Gebüschen, ranunculoides in schalligen Wäldern. Adonis aestivalis unter der Saat. Ranunculus aquatilis in stehenden Gewässern, Flammula auf Sumpfwiesen der Ebenen und Berge, Ficaria, acris, repens gemein, lanuginosus, polyanthemos auf sonnigen Triften, auricomus in schattigen Wäldern, arvensis an cultivirten Orten. Callha palustris, Helleborus viridis. In den Bergwäldern zwischen dem Kalvarienberge und Zitnya. Isopyrum thalictroides in Wäldern beim Zitnya. Nigella arvensis selten auf Aeckern, Aquilegia vulgaris am Kalvarienberge. Delphinium Consolida. Actaea spicata in Wäldern um den Zitnya.

Pavaper Rhoeas, Chelidonium majus, gemein, Fumaria officinalis an cultivirten Orten, Corydalis cava, solida in Wäldern. Nasturtium silvestre, Barbarea vulgaris auf Triften. Turritis glabra in Wäldern. Arabis liirsuta, arenosa auf Triften. Cardamine amara, pratensis auf Wiesen. Dentaria bulbifera in schattigen Wäldern. Sisymbrium officinale, Sophia, Alliaria gemein. Sinapis arvensis, Alyssum calycinum, Farsetia incana, Draba verna, innerhalb Schemnitz häufig. Thlaspi arvense auf Aeckern, perfoliatum an Bächlein. Lepidium campestre an Ackerrändern. Capsella bursa pastoris, Neslia paniculata häufig auf Aeckern. Raphanus Raphanistrum.

Helianthemum vulgare auf allen sonnigen Triften.

Viola arvensis an kultivirten Orten, odorata, canina, hirta, tricolor in schattigen Bergwäldern.

Reseda lutea, luteola innerhalb Schemnitz zerstreut. Wiesen.

Polygala vulgaris auf trockenen Triften, amara auf nassen

Gypsophyla muralis im Sande der Wassergräben.

Silene nutans, inflata am Kalvarienberge. Dianthus deltoides, Armeria am Kalvarienberge, Carthusianorum, Agrostemma Githago unter der Saat.

Lychnis floscuculi auf Sumpfwiesen, viscaria auf grasreichen Bergtriften.

Spergula arvensis auf Aeckern. Lepigonum rubrum im Sande der Wassergräben. Moehringia trinervia auf feuchten. Stellen des Kalvarienberges. Stellaria Holostea in Bergwäldern, graminea auf Sumpfiwiesen, media, Malachium aquaticum an Wassergräben. Cerastium arvense, Linum catloarticum auf allen Wiesen. Malva rofundifolia gemein.

1) Linnaea 1863. 
Hypericum hirsutum in Bergwäldern," perforatum, Geranium phaeum, silvaticum in Wäldern, pratense auf Sumpfwiesen. Robertianum und sanginineum am Kalvarienberge.

Acer campestre, Pseudoplatanus, tataricum, in Bergwäldern.

Impatiens nolitangere an allen Bergbächen.

Oxalis Acetosella in schattigen Waldungen.

Evonymus europaeus, verrucosus in Bergwäldern. Rhamnus carthartica, Frangula ebendaselbst. Genista germanica, tinctoria auf sonnigen Triften. Cytisus nigricans in schattigen Bergwäldern. Ononis spinosa, Anthyllis vulneraria am Kalvarienberge. Medicago lupulina, Melilotus officinalis, Trifolium pannonicum, pratense, ochroleucum, medium auf höheren Triften, arvense auf Aeckern. Lotus corniculalus, Astragalus glycyphyllos in Wäldern gegen Zitnya. Vicia lathyroides, pisiformis, silvatica in Wäldern und Gebüschen, pannonica, Cracca, villosa auf Aeckern und Wiesen. Lathyrus pratensis auf Wiesen, latifolius, silvestris auf höheren Bergtriften. Orobus vernus in schattigen Wäldern. Prunus spinosa, Spiraea Aruncus, Vlmaria an Lachen und Sumpfwiesen. Geum urbanum am Kalvarienberge: Fragaria vesca, collina auf trockenen Triften. Rubus caesius gemein, fruticosus, Idaeus in Bergwäldern. Potentilla rupestris am Zitnya, recta am Kalvarienberge, alba an Waldrändern, anserina, argentea, reptans, Tormentilla, verna auf Wiesen. Rosa canina, spinosissima in Wäldern gegen Zitnya. Alchemilla vulgaris an Waldrändern. Poterium Sanguisorba auf Triften.

Crataegus oxyacantha gemein, Coloneaster vulgaris Lindl. am Zitnya, Sorbus Aucuparia, Aria. In den Bergwäldern Pyrus sylvestris.

Epilobium angustifolium, palustre, parviflorum auf Wiesen, montanum in Bergwäldern, Circaea lutetiana in schattigen Bergwäldern. Gewässern.

Callitriche vernalis in stehenden und langsam fliessenden

Lythrum Salicaria, virgatum auf Wiesen.

Bryonia alba, dioica an Zäunen und Hecken.

Scleranthus annuus auf Aeckern.

Sedum acre auf dürren Hügeln, Telephium am Kalvarienberge, Sempervivum tectorum auf Mauern.

Ribes Grossularia in schattigen Wäldern.

Saxifraga Aizoon am Zitnya, granulata anf Triften.

Chrysosplenium alternifolium an Bergbächen.

Sanicula europaea in schattigen Wäldern, Eryngium campestre. Carum carvi auf Wiesen. Pimpinella Saxifraga, Falcaria Rivini, Bupleurum rotundifolium unter der Saat. Oenanthe Phellandrium und Angelica silvestris an Wassergräben. Pastinaca safiva, Heracleum Sphondyfium, Tordylium maximum, Siler trilobum in den Wäldern $z$ wischen dem Kalvarienberge und Zitnya. Anthriscus Cerefolium, silvestris in Gebüschen. Conium maculatum, Hedera Helix. Blühende Stämme bei Hodris. 
Cornus mas und sanguinea in Bergwäldern.

Viscum album aufBirnbäumen. Loranthus europaeus auf Eichen.

Adoxa moschatellina in Gebüschen der Wälder. Sambucus racemosa in Wäldern, Ebulus, nigra gemein. Viburnim Lantana an Waldrändern. Opulus auf Sumpfwiesen.

Lonicera Xylosteum und nigra in höheren Wäldern. Asperula odorata in Wäldern, cynanchica und galioides M. B. auf Triften und in Gebüschen.

Galium silvaticum in Wäldern, cruciatum, Mollugo, verum auf Triften, uliginosum auf Sumpfwiesen. Valeriana officinalis, dioica, tripteris in schattigen Bergwälderu. Valerianella olitoria auf höhern Triften. Dipsacus silvestris, Knautia arvensis, Scabiosa ochroleuca. Ueberall gemein.

Eupatorium cannabinum an sumpfigen Orien, Tussilago Farfara, Petasites officinalis, alba an Bergbächen. Bellis perennis auf Wiesen. Erigeron acris et $\beta$. serotinus auf trockenen Triften, canadensis, Solidago virga aurea in Bergwäldern. Bidens cernua, tripartita an sumpfigen Orten. Inula salicina, Filago arvensis gemein. Gnaphalium silvaticum, dioicum in Bergwäldern, uliginosum auf Sumpfwiesen. Artemisia Absynthium, vulgaris, Achillea Millefolium, Anthemis Colula, arvensis gemein, tinctoria am Kalvarienberge. Matricaria Chamomilla, Chrysanthemum inodorum, Leucanthemum auf Wiesen und Aeckern, corymbosum in Bergwäldern. Senecio vulgaris gemein, erucifolius, Jacobaea auf Triften und Wiesen, silvaticus in Bergwäldern, viscosus in Holzschlägen, nemorensis in Gebüschen der Ehene. Cirsium eriophorum, arvense, gemein, oleraceum an Wassergräben. Carduus acanthoides, Lappa major, minor gemein. Carlina acaulis aufTriften, vulgaris, in Holzschlägen. Serratula tinctoria in Gebüschen. Centaurea montana, phrygia, Scabiosa auf höheren Triften, Cyanus, Lapsana communis in Gebüschen Cichorium Intybus. Leontodon autumnalis auf Wiesen. Pioris hieracioides, Tragopogon pratensis. Hypochoeris maculata auf höhern Bergtriften. Phoenixopus purpureus, Lactuca muralis in Wäldern, scariola saligna auf Aeckern und buschigen Orten. Crepis foetida, setosa an Ackerrändern, tectorum, Hieracium Pilosella, Auricula auf Triften, prenanthoides, umbellatum in Wäldern. Xanthium strumarium und spinosum an Aeckern.

Phyteuma spicatum in schattigen Wäldern, orbiculare auf höhern Triften. Campanula persicifolia, glomerata am Kalvarienberge, Trachelium in Wäldern, patula auf Wiesen.

Vaccinium Myrtillus auf allen höheren Triften. Monotropa Hypopitys in schattigen Bergwäldern. Cynanchum Vincetoxicum in Gebüschen.

Vinca minor in Bergwäldern.

Gentiana cruciata, Amarella, Erythraea Centaurium auf höheren Triften. Convolvulus arvensis, sepium, Cuscuta europaea Asperugo procumbens auf Schutthaufen. Anchusa officinalis, Symphytum officinale, tuberosum, auf Wiesen und Triften. Pulmonaria 
offecinalis, in Wäldern, mollis, angustifolia an ähnlichen Orten und in Gebitschen. Lithospermum arvense, Myosotis palustris an sumpfigen stellen, hispida, silvatica auf höheren Triften. Solanum Dulcainara in Gebüschen an Wassergräben, nigrum, Atropa Belladonna in Bergwäldern. Hyoscyamus niger, Verbascum nigrum, phoeniceum auf höheren Triften, Thapsus. Scrophularia nodosa an Bächen. Digitalis grandiflora $\mathrm{L}$ a m. in Bergwäldern. Linaria genistaefolia, auf sonnigen Triften, vulgaris, Veronica Chamaedrys, spicata, prostrata, verna auf grasreichen Triften, officinalis in Wäldern, arvensis, agrestis, triphyllos, hederaefolia auf Aeckern. Beccabunga, serpyllifolia an sumpfigen Orten.

Melampyrum arvense an Waldrändern, nemorosum am Kalvarienberge. Rhinanthus major, minor auf Wiesen. Euphrasia Odontites, officinalis, auf Wiesen und Triften.

Salvia verticillata, pratensis auf Triften, silvestris, Origanum vulgare in Bergwäldern, Lycopus europaeus, Mentha aquatica, silvestris an Wassergräben. Clinopodium vulgare, Thymus serpyllum, Calamintha Acinos auf Triften. Nepeta Cataria vereinzelt an Zäunen. Glechoma hirsuta in Bergwäldern, hederacea. Melitis Melissophyllum in Bergwäldern. Lamium maculatum, purpureum, amplexicaule, Galeobdolon luteun in Wäldern. Galeopsis Ladanum, Tetrahit, Stachys alpina, silvestris in Wüldern, germanica auf Wiesen, annua, Betonica officinalis auf sonnigen Triften. Ballota nigra, Leonurus Cardiaca, Scutellaria hastifolia an Wassergräben. Prunella alba auf Triften, vulgaris, Ajuga reptans auf Wiesen, genevensis in Wäldern. Teucrium Chamaedris, Verbena officinalis, Lysimachia vulgaris in Gebüschen., Nummularia, Anagallis arvensis, Primula officinalis, elatior, acaulis in Wäldern. Plantago major, media, lanceolata, Amaranthus retroflexus. Chenopodium bonus Henricus, album, Vulvaria, auf Schuthanfen. Atriplex nitens an Zäunen. Rumex acetosa, crispus auf Triften, Acetosella in Waldern. Polygonum Convolvulus auf Aeckern, aviculare gemein. Daphne Mezereum in Wäldern gegen den Zitnya hin.

Asarum europaeum in Bergwaldern.

Thesium intermedium auf Bergtriften.

Euphorbia amygdaloides in Wäldern. Esula, helioscopia, Peplus auf Brachäckern, Cyparissias, Mercurialis perennis in Bergwäldern, annua, Urtica urens, dioica. Parietaria erecta auf Schutthaufen. Humulus Lupulus, Vlmus campestris, Fagus silvatica. Quercus pedunculata, Robur, Corylus Avellana, Carpinus Betulus in Waldern.

Salix alba, Capraea, fragilis, viminalis an feuchten Stellen. Populus tremula in Wäldern. Betula alba in Wäldern. Alnus glutinosa an feuchten Orten. wäldern.

Juniperus communis, Pinus silvestris, Abies excelsa in Berg-

Alisma Plantago an Wassergräben,

Lemna minor in stehenden Gewässern.

Sparganium ramosum ebendaselbst. 
Arum maculatum in schattigen Bergwäldern.

Orchis sambucina $\beta$. incarnata W., Morio, globosa auf Bergtriften. Habenaria viridis an feuchten Bergwiesen. Gymnadenia conopsea, auf sumpfigen Wiesen hinter dem Kalvarienberge. Platanthera bifolia, Cephatanthera pallens, rubra in Bergwäldern. Neottia nidusavis in Wäldern bei St. Antal. Listera ovata am Zitnya.

Iris graminea auf Wiesen.

Galanthus nivalis am Koselnik.

Paris quadrifolia in schattigen Wäldern.

Concallaria majalis, multiflora, Polygonatum, verticillata, Majanthemum bifolium in schattigen Bergwäldern.

Lilium Martagon in Bergwaldungen. Ornithogalum nutans, umbellatum, pyrenaicum auf Triften. Gagea lutea in schattigen WäLdern, pusilla auf Wiesen. Scilla bifolia im Rother-Brunnen-Walde. Allium flavum an felsigen Orten. Muscari comosum auf Feldern, racemosum auf grasreichen Triften.

Colchicum autumnale auf Wiesen.

Juncus effusus, lamprocarpus auf Wiesen, bufonius an sandigen Orten.

Luzula albida, campestris in Bergwäldern.

Scirpus silvaticus in Gebüschen, palustris an sumpfigen Orten. Eriophorum angustifolium. Auf allen feuchten Wiesen. Carex praecox, pilosa, silvatica in Wäldern, Schreberi, acuta, filiformis, hirta auf Wiesen.

Andropogon Ischaemum, Panicum crus Galli, Anthoxanthum. odoratum auf Triften. Alopecurus pratensis, geniculatus an Wassergräben. Setaria glauca, Phleum pratense, Phalaris arundinacea an Wassergräben. Milium effusum auf Wiesen. Koeleria cristata, Aira caespitos a ebendaselbst. Holcus lanatus, mollis auf Triften. Melica nutans, uniflora in Wäldern. Briza media auf allen Triften. Poa annua, pratensis, Eragrostis, trivialis auf Wiesen und Aeckern, compressa auf Triften, nemoralis in Wäldern. Glyceria fluitans an Wassergräben. Dactylis glomerata, Festuca ovina, Bromus mollis, tectorum, Hordeum murinum, Lolium arvense auf Feldern. Nardus stricta auf höheren Triften.

Nach einem fünftägigen Aufenthalte verliess ich die Stadt mit dem Wunsche, es möge mir vergönnt sein, dieselbe noch ofters besuchen zu liönnen.

Ich nahm meinen Weg gegen Hodris. Zu beiden Seiten der Strasse und auf dem nahe gelegenen Gebirge sah ich: Alchemilla vulgaris, Anthriscus silvestris, Arabis hirsuta, arenosa, Arenaria serpyllifolia, Asarum europaeum, Aspidium Filix mas, Asplenium Trichomanes, Bellis perennis, Briza media, Campanula Trachelium, Carlina vulgaris, Cardamine Impatiens. Im Innern eines Quellenganges; Centaurea Scabiosa, Circaea alpina, Clematis Vitalba, Cornus sanguinea, Crepis setosa, Cynanchum Vincetoxioum, Cynoglossum officinale, Dianthus delloides, Digitalis grandiflora, Epilo- 
bium palustre, Erigeron acris, Galeobdolon luteum, Geranium columbinum et Robertianum, Glyceria fluilans, Gnaphalium dioicum, silvaticum, Hieracium Auricula, vulgatum, Hypericum hirsutum, Impatiens noli tangere, Inula Conyza, Lapsana communis, Laserpitium trilobum, Lonicera Xylosteum, Lazula albida, Majanthemum bifolium, Melampyrum nemorosum, Moehringia trinerviä, Origanum vulgare; Oxalis Acetosella, Polygonum Convolvulus, Polypodium Dryopteris, Potentilla Tormentilla, Ranunculus bulbosus, Rumex Acetosella, Sagina procumbens, Saxifraga granulata, Sorophularia aquatica, Sedum acre, Telephium, Senecio viscosus, Silene nutans, Sorbus Aucuparia, Spergula arvensis, Spivaea Aruncus, Stellaria graminea, Thlaspi perfolialum, Thymus Acinos, Vaccinium MIyrtillus, Verbascum nigrum, Veronica officinalis, Euphorbia amygdaloides, Lactuca murulis und Monotropa Hypopitys.

Von Hodris bis zum Meierhofe nächst der Brücke, die nach Zsarnovics führt, traf ich noch folgende Gewächse an: Alyssum calycinum, Aquilegia vulgaris verwildert, Artemisia Absynthium, Campanula patula, Chaerophyllum aromaticum, Chenopodium bonus thenricus, Chrysanthenum corymbosun, Cirsium oleraceum, Cuscuta europaea, Cystopteris fragilis, Dactilis glomerata, Erysimum Alliaria, Galiım uliginosum, Geranium pleaeum, Gnaphalium uliginosum, Juncus eff usus, bufonius, lamprocarpus, an Bergbächen. Lamium maculatum, Lathyrus silvestris, Lepidium campestre, an Wegen. Lepigonum rubrum und Lycopus exaltatus, an Bergbächen. Marrubium peregrinum, Petasites officinalis, am Bergbache. Pulicaria vulgaris, Salvia verticillata, Saponaria officinalis, Silene inflata, Siler trilobum, Spiraea Filipendula und ulmifolia, Veronica officinalis, Vicia sepium, Viola arvensis, tricolor, Xanthium spinosum, spärlich.

Von hier der Gran entlang bis Lót sah ich: Alnus glutinosa, Althaea officinalis, Artemisia Absynthium, Aristolochia Clematitis, auf Feldern. Arenaria serpyllifolia, Cirsium canum, Cyperus fuscus, Epilobium palustre, Galega officinalis, Herniaria glabra, Juncus bufonius, lamprocarpus, Lathyrus pratensis, Lepigonum rubrum, Linum catharticum, Lythrum hyssopifolium, Malachium aquaticum, Menyanthes trifoliata auf Rohrgrund. Myriophyllum verticillatum, in der Gran. Nasturtiun palustre, Orobanche ramosa, auf Hanffeldern. Potentilla supina, Ranunculus fluitans, in der Gran. Flammula, auf Rohrgrund, sceleratus, bulbosus, Sagina procumbens, Salix canescens Sanguisorba officinalis, auf Wiesen. Scirpus acicularis, maritimus, Silene inflata, nutans, Solanum Dulcamara, Trifollum filiforme. Alopecurus geniculatus, Armoracia rustica, in Bauerngärlen. Linaria minor und Limosella aquatica.

Zwischen Lót und Rudnó erblickte ich: Campanula Trachelium, Epilobium angustifolium, Juncus effusus und Juniperus communis.

Von Rudnó bis zur Brücke nach Königsberg sah ich bloss Agrimonia Eupatoria, Althaea officinalis, Reseda luteola und Xanthium spinosum. 
Doch weit interessanter als die Wiesen bei Szänto waren die gegen Königsbero hin, an jenseitigen Ufer der Gran. Hier traf ich Pflanzen an, die ich bisher noch nirgends bemerkte : Aegopodium Podagraria, Agrimonia Eupatoria, Betonica officinalis, Campanula glomerata, Carex hirta, Carlina vulgaris, Chenopodium polyspermum, Cirsium canum, Convällaria majalis, in Gebüschen. Cornus sanguinea, Crepis foetida, Cytisus nigricans, Dianthus Carthusianorum, deltoides, Epilobium palustre, angustifolium, Eupatorium cannabinum, Galium rubioides, Genista tinctoria, Geranium pratense, Hieracium umbellatum, Inula germanica, in Gebüschen. Juncus lamprocarpus, compressus, Lapsana communis, Lavatera thuringiaca, Leersia oryzoides, Lepigonum rubrum. Lysimachia oulgaris, Melampyrum nemorosum, Oenothera biennis, in Gebüschen. Ononis hircina, Panicum sanguinale. Polypodium Filixmas, Ranunculus Flammula, Rubus fruticosus, Sanguisorba officinalis, Sempervivum hirtum, auf den benachbarten Felsen. Senecio viscosus, Setaria italica, Silene inflata, Solidago virga aurea, Succisa pratensis, Teucrium Scordium, Thalictrum flavum wurden hier eingelegt.

Bei Königsberg besuchte ich die Ufer der Gran und fand daselbst: Arenaria serpyllifolia, Cyperus fuscus, Gnaphalium uliginosum, Gypsophila muralis, Herniaria glabra, Juncus bufonius, effusus, lamprocarpas, Lepigonum rubrum, Limosella aquatica, Linum catharticum, Lythrum hyssopifolium, Sagina procumbens und Xanthium spinosum.

Weiterhin auf dem Wege durch die Wiesen nach St. Benedek sah ich: Achillea nobilis, Alisma Plantago, Alepocarus geniculatus, an sumpfigen Orten. Bidens cernua, Callitriche vernalis, in Sümpfen. Carlina vulgaris, Clematis Vitalba, an Hecken. Eragrostis poaeoides. Euphrasia officinalis, Glyceria fluitans und spectabilis, an den Sümpfen. Lathyrus pratensis, tuberosus, Lysimachia vulgaris, Malachium aquaticum, Mentha sylvestris. Myriophyllam verticillatum und spicatum, an den Sümpfen. Oenothera biennis, Phalaris arundinacea, Potamogeton crispus, in den Sümpfen. Scirpus palustris, acicularis, an den Sümpfen. Sanguisorba officinalis, Silene inflata, Sparganium simplex, in den Sümpfen. Succisa pratensis, Verbascum Blattaria, Viburnum Opulus und Ranunculus Flammula.

Von St. Benedek bis Némethy legte ich ein: Achillea nobilis, Artemisia Absynthium, Briza media, Centaurea Scabiosa, Chaiturus Marrubiastrum, Chondrilla juncea, Erysimum canescens, Lepidium canpestre, Marrubium peregrinum, Nigella anyensis, Ranunculus bulbosus. An angeschwemmten Orten. Reseda luteola. Stachys germanica und Verbena officinalis.

Von Némethy bis Csarád fand ich: Althaea officinalis, Bryonia alba, Chaiturus Marrubiastrum, Ly thrum hyssopifolium und Verbascum Blattaria.

Zwischen Csarád und Aranyos Maroth kamen an der Strasse und in den nahe gelegenen Wäldern vor: Artemisia Absynthium, Carlina vulgaris. Dianthus Armeria, Dorycnium herbaceum, Echi- 
nosperimum Lappula, Erigeron acris, Erythraea pulchella, Euphrasia Odontiles, auf Wiesen. Farsetia incana, Lactuca saligna, Rubus caesius, Sisymbriun officinale und Tussilago Farfara, auf der Strasse.

Von Aranyos Maroth bis Hizér ist die Flora auf die Gräben beschränkt, daher unbedeutend.

Yon Hizér bis Choes traf ich an Ackerrändern, ausgetrockneten Wassergraben und auf Wiesen an: Artemisia scoparia, Campanula patula, Cephalaria transsilvanica, Cerastium arvense, Erythraea pulchella, Euphrasia Odontites, Eragrostis megastachya, Gnaphalium uliginosum, Gypsophila muralis, Heliotropium europaeum, Juncus lamprocarpus, bufonius, Kochia arenaria, Lactuca saligna, Lapsana communis, Lepidium ruderale, Lepigonum rubrum, Lythrum hyssopifolium, Nigella arvensis, Nonnea pulla, Passerina annua, Ranunculus bulbosus, Saponaria officinalis, Scleranthus annus, Tanacetum vulgare, Thalictrum flavum, Trifolium arvense und fliforme, Triglochin palustre.

Zwischen Chocs und Gross-Herestény kamen vor auf Feldern und Wiesen: Asparagus officinalis, Bellis perennis, Euprasia Odontites, Juncus effusus und Scleranthus annuus.

Von Gross-Herestény bis zum Jägerhause im Családer Walde fand ich an Ackerrändern und Wiesen: Adonis aestivalis, Alopecurus geniculatus, Astragalus Cicer, Caduus nutans, Galega officinalis. Juncus effusus, Lactuca saligna, Lathyrus pratensis, Linaria Elatine, Lotus tenuifolius, Scirpus silnaticus, Sparganium ramosum und Verbascum Blattaria.

Im Családer Walde waren zerstreut: Adonis vernalis, Anthericum ramosum, Campanula glomerata, Chrysanthemum corymbosum, Cytisus nigricans, Dorycnium herbaceum, Genista tinctoria und Serratula tinetoria.

Von hier wählte ich den kürzesten Weg, und nach einer halben Stunde langte ich wohlbehalten in Lapas-Gyarmath an.

Wien, im Oktober 1863.

\section{Versuche über die Keimfähigkeit alter Sämereien.}

Von Godwin Böckel.

Es lässt sich wohl mit Bestimmtheit bei keiner Pflanzengattung genau angeben, wie alt der Samen werden darf, ohne seine Keimkraft zum Theil oder gänzlich zu verlieren, jedoch nach meinen Versuchen habe ich mich überzeugt, dass sebr oft Samen weggeworfen werden als vollkommen unkeimfähig, die auf passende Weise behandelt noch viele gesunde Pflanzen hätten erzeugen können, indem die Keimfähigkeit keineswegs erstorben, sondern, wenn ich so sagen darf, nur ein- 